九州大学学術情報リポジトリ

Kyushu University Institutional Repository

\title{
Automated segmentation method of white matter and gray matter regions with multiple sclerosis lesions in MR images
}

Magome, Taiki

Japan Society for the Promotion of Science

Arimura, Hidetaka

Department of Health Sciences, Faculty of Medical Sciences, Kyushu University

Kakeda, Shingo

Department of Radiology, University of Occupational and Environmental Health, School of Medicine

Yamamoto, Daisuke

Department of Health Sciences, Graduate School of Medical Sciences, Kyushu University

他

http://hdl. handle. net/2324/25450

出版情報: Radiological Physics and Technology. 4 (1), pp.61-72, 2011-01. Springer Japan バージョン :

権利関係: (C) Japanese Society of Radiological Technology and Japan Society of Medical Physics 2010 


\section{Automated segmentation method of white matter}

\section{2 and gray matter regions with multiple sclerosis}

\section{3 lesions in MR images}

4

5

6

7

8

9

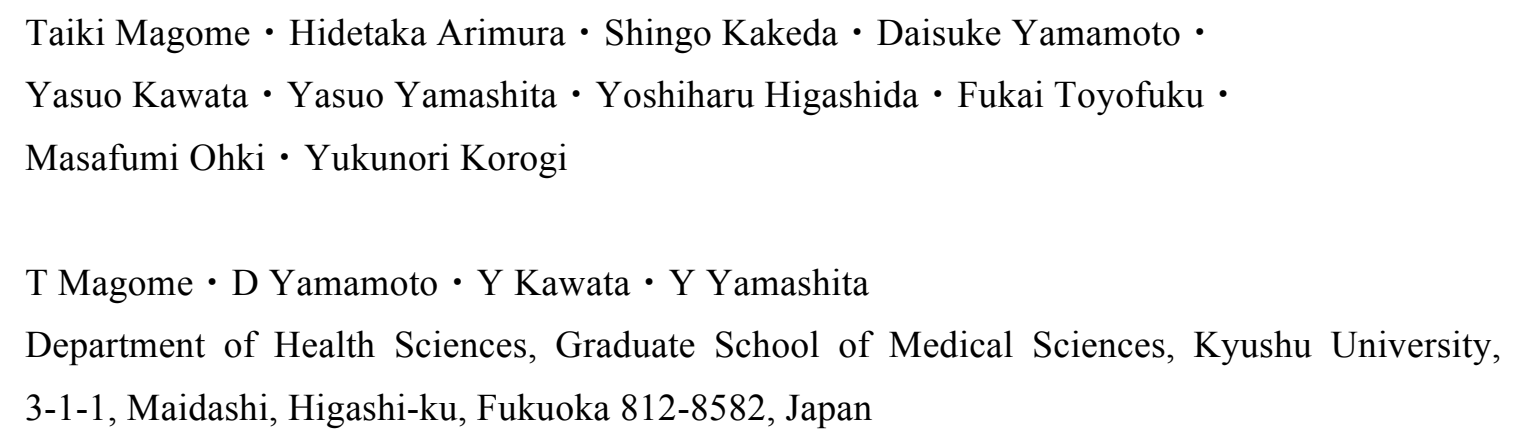




\section{$35 \quad$ Abstract}

36 Our purpose in this study was to develop an automated method for segmentation of white matter 37 (WM) and gray matter (GM) regions with multiple sclerosis (MS) lesions in magnetic resonance (MR) images. The brain parenchymal (BP) region was derived from a histogram analysis for a T1-weighted image. The WM regions were segmented by use of addition of MS candidate regions, which were detected by our computer-aided detection system for the MS lesions, and subtraction of a basal ganglia and thalamus template from "tentative" WM regions. The GM regions were obtained by subtraction of the WM regions from the BP region. We applied our proposed method to T1-weighted, T2-weighted, and fluid-attenuated inversion-recovery (FLAIR) images acquired from 7 MS patients and 7 control subjects on a $3.0 \mathrm{~T}$ MRI system. The average similarity indices between the specific regions obtained by our method and by neuroradiologists for the BP and WM regions were $95.5 \pm 1.2 \%$ and $85.2 \pm 4.3 \%$, respectively, for MS patients. Moreover, they were $95.0 \pm 2.0 \%$ and $85.9 \pm 3.4 \%$, respectively, for the control subjects. The proposed method might be feasible for segmentation of WM and GM regions in MS patients.

50 
Multiple sclerosis (MS) is a neurological disorder in the central nervous system. The progressive development of brain atrophy is a well-known characteristic of MS and is viewed as a potential marker of brain damage [1-7]. Therefore, the quantitative evaluation of brain atrophy is important for diagnosis or follow-up of MS by use of magnetic resonance (MR) imaging. Previous MR studies have shown that white matter atrophy in patients with primary progressive MS was closely related to clinical outcome [8], and gray matter atrophy in relapsing-remitting MS (RRMS) was related to the Expanded Disability Status Scale [9]. In such studies, neuroradiologists need to segment the brain parenchyma into the white matter and gray matter regions. However, it is laborious for neuroradiologists to determine the atrophies of the white matter and gray matter regions in MR images on a slice-by-slice basis. Therefore, a number of semi-automated and automated methods for segmentation of the white matter and gray matter regions have been developed for assisting radiologists in evaluating the atrophies of these regions in clinical practice [10-19]. However, these methods were developed for MR images without MS lesions. Ge et al. [2] proposed a semi-automated method based on fuzzy connectedness [10] for segmentation of white matter and gray matter regions with MS lesions. Stefano et al. [5] developed a semi-automated method by using the SIENA (structural imaging evaluation of normalized atrophy) X software [20] for segmentation of cortical gray matter regions with MS lesions.

In general, semi-automated methods are time-consuming, and the results depend on how the methods are used. Alfano et al. $[11,21]$ developed an automated method for extraction of the white matter and gray matter regions in MS patients for estimation of their atrophy, although they did not evaluate the accuracy of their segmentation method. Many researchers employed statistical parametric mapping (SPM99) [12] as an automated segmentation tool for 
the white matter and gray matter regions [3,6-9]. However, a majority of MS lesions in the white matter regions were misclassified as gray matter or cerebrospinal fluid (CSF) by SPM99. Therefore, further studies are still required for automatically segmenting the white matter and gray matter regions including MS lesions for more accurate evaluation of the atrophies in both regions in MS patients. That is because the atrophy in the white matter regions cannot be correctly evaluated if the MS lesions in the white matter regions are not included. Our purpose in this study was to develop an automated method for segmentation of the white matter and gray matter regions including MS lesions in MR images.

\section{Materials and methods}

\subsection{Clinical cases}

The MR images of seven patients with RRMS and seven normal controls were used for this study. Patients who were diagnosed as having MS and had MR examinations from January 2007 to April 2008 were sequentially selected. Non-MS subjects, who were matched with the MS patients in terms of age and gender, were chosen as control cases from all non-MS patients who had MR examinations from January 2007 to March 2007. The MS group (5 females and 2 males) had a mean age of 31 years (range: $24-51$ years), and the control group (5 females and 2 males) had a mean age of 30 years (range: 15-56 years). This study was performed under a protocol approved by the institutional review board of the university hospital.

All brain MR images were acquired with a 3.0 Tesla MR system (Signa Excite; GE Medical systems, Milwaukee, Wis, USA). The following three imaging parameters were used: 2500/9.1/1000/224×320/2/1 minute 30 seconds [repetition time msec/echo time msec/inversion 

msec/echo time msec/matrix size/NEX/echo-spacing/imaging time) for each 2D T2-weighted fast spin-echo imaging, and 12000/140/2600/224×256/2/9.1/3 minutes 20 seconds (repetition time msec/echo time msec/inversion time/matrix size/NEX/echo-spacing/imaging time) for each 2D fluid-attenuated inversion-recovery (FLAIR) imaging. All images were acquired with a section thickness of $5 \mathrm{~mm}$, an intersection gap of $1 \mathrm{~mm}$, a field of view of $22 \mathrm{~cm}$, and 16-bit gray levels. Zero-fill interpolation processing was used for reconstruction of $2 \mathrm{D}$ images with $512 \times 512$ pixels and a pixel size of $0.4297 \mathrm{~mm}$, which result in apparent high resolution images. selected per case, because Carone et al. [7] had reported that the basal ganglia, thalamus, and superior cortex were atrophied in MS patients. analysis of a gray-level histogram [22]. Figure 1 shows an example of a histogram of an original T1-weighted image. The histogram of a T1-weighted image can be divided into four parts, i.e., background, CSF, brain parenchymal, and fat regions. First, the head region was extracted from the original T1-weighted image by use of the threshold value $T_{B G}$ given by where $M_{B G}$ and $S D_{B G}$ are the mean value and the standard deviation (SD), respectively, determined from the first largest peak (the first left peak) with more than a certain number of pixels in the histogram as shown in Fig. 1, which was empirically set as 10,000 pixels in this 
study. $k_{\mathrm{BG}}$ is a constant. Second, the CSF regions were removed from the head region with the threshold value $T_{C S F}$ obtained by an automated thresholding technique based on linear discriminant analysis [23] for the histogram of the T1-weighted image. Third, the brain parenchymal region was extracted by reduction of the fat regions in a manner similar to that for the background. The threshold value for the fat region $T_{F A T}$ was determined by

$$
T_{F A T}=M_{B P}+k_{B P} S D_{B P},
$$

where $M_{B P}$ and $S D_{B P}$ are the mean value and the standard deviation, respectively, obtained from the second largest peak (the first right peak) with more than a certain number of pixels in the histogram of the brain parenchymal and fat regions as shown in Fig. 1, which was empirically set as 700 pixels in this study. $k_{\mathrm{BP}}$ is a constant. Note that some small holes could occur within the brain parenchymal region, because a number of pixels in the brain parenchymal region are similar to those in the fat regions. Therefore, the holes were filled in by the addition of a rough brain parenchymal region without holes to the brain parenchymal region after reduction of the fat region. The rough brain parenchymal region without holes was obtained by application of a circular morphological erosion kernel to the head region. In this study, the constant values $k_{\mathrm{BG}}$ and $k_{\mathrm{BP}}$ were set as 10 and 4 , respectively. The parameters were determined empirically by use of nine clinical cases in the study of Kawata et al. [22], which are different from cases used in this study.

\subsection{Segmentation of white matter regions}

Figure 2 shows the overall scheme for segmentation of the white matter regions. First, a brain parenchymal region was segmented by use of the method mentioned above. Second, a T2-T1 subtraction image was obtained by subtraction of a T1-weighted image from a T2-weighted image. Third, the "tentative" white matter regions were segmented on the 
subtraction image by use of a level set method [24] in the brain parenchymal region. Fourth, MS candidate regions detected by our computer-aided detection (CAD) system [25] were added onto the "tentative" white matter regions. Fifth, the white matter regions were determined by removal of a basal ganglia and thalamus (BGT) template from the white matter regions.

\subsubsection{Subtraction image between T2-weighted and T1-weighted images} contrast between the white matter and gray matter regions. Figure 3 shows the three pixel value histograms of the brain parenchymal region in T2-weighted, T1-weighted, and subtraction images, whose pixel values were normalized from 0 to 1023 . In the T2-weighted image, the white matter regions have lower pixel values compared with the gray matter regions, whereas there is an inverse relationship in the T1-weighted image. On the other hand, the contrast could not be detected in the T1-weighted images, because the peaks of the white matter and gray matter regions were overlapped. The average contrast between the peak pixel values of the white matter and gray matter regions for 14 slices selected from 14 cases was $174 \pm 53.7$ pixel values for the subtraction images, and $119 \pm 21.3$ pixel values for the T2-weighted images. As a result, the white-matter-gray-matter contrast in the subtraction image was higher than that in the T2-weighted image with a statistically significant difference $(P<0.01)$. Therefore, the contrast between the white matter and gray matter regions was increased by subtraction of the T1-weighted image from the T2-weighted image. Figure 4 shows the brain parenchymal regions in three images, i.e., T2- weighted, T1-weighted, and the T2-T1 subtraction image. The T2-T1 subtraction image seems to have the highest contrast. 

image by use of an automated thresholding technique based on a linear discriminant analysis and long fat regions as well as the small white matter regions were still remained. Therefore, two types of candidate regions were selected as the white matter regions. One type was the candidate region of the largest size, and the other type was a region whose mean pixel value was within the range between the mean pixel value \pm a SD of the largest region. Finally, a morphological erosion operation with a $3 \times 3$ kernel was applied three times to the binary image with white matter candidate regions on the assumption that the eroded regions could be inside the "true" white matter regions. The resulting white matter candidate regions were considered as initial white matter regions.

\subsubsection{Segmentation of "tentative" white matter regions based on a level set method}

The "tentative" white matter regions were segmented based on a level set method, where a new speed function was developed in this study for accurate segmentation of white matter regions. The level set method is an active contour model, which has been widely used for segmentation of some anatomical regions in medical images such as brain regions in MR images $[24,26,27]$. In our research, the level set method was performed by means of a fast narrow band method $[28,29]$ for reducing the calculation time. First, a level set function $\phi$ was determined as a signed distance function from the contour of the initial white matter regions, according to the following partial differential equation: 
203 where $t$ is the time, $F$ is the speed function, and $\nabla$ is the gradient operator. While the level set 204 function is updated, the zero level set $(\phi=0)$ moves according to the speed function in the 205 three-dimensional (3D) level set function. Here, the zero level set is called a "moving front". 206 Finally, the update of the level set function was stopped if a certain ratio of pixels $r_{t}$ on the zero 207 level did not move within a certain number of iterations $i_{t}$. The zero level $(\phi=0)$ of the function is considered as the final contour of the object. In this study, we developed a speed function $F$ given by

$$
F=b(v+\rho \kappa),
$$

211 where $b$ is the edge indicator function, $v$ and $\rho$ are constants, and $\kappa$ is the mean curvature. The 212 term of $\rho \kappa$ gives the smoothness of the front propagation. The edge indicator function $b$ is 213 defined as

$214 \quad b=\frac{1}{1+|\nabla I(x, y)|}$,

where $I(x, y)$ is the image processed with an adaptive partial median (APM) filter [30]. The edge indicator function $b$ plays an important role for stopping the moving front propagation at

217 the desired boundary of the object, because the function $b$ approaches zero when the moving 218 front arrives at the object boundary. However, if the object boundary includes noise, the segmentation result would be inaccurate and unstable. Therefore, some smoothing filter such as a Gaussian filter should be applied to the original image for reduction of noise prior to application of the level set method. However, an edge-preserving smoothing (EPS) filter would be preferred as a smoothing filter, because the general smoothing filters blur the edge of an object. In this study, we chose an APM filter developed by Lee et al. [30] as an EPS filter, because the APM filter can reduce noise with preserving edges owing to their adaptive filter 
images obtained by three smoothing filters, i.e., the Gaussian filter, an EPS filter [31], and the

APM filter. The image processed with the APM filter seems to be the best among the three images in terms of reducing noise and preserving edges. In this study, the parameter values $r_{t}, i_{t}$, $v$, and $\rho$ were set as $0.999,500,1.0$, and -0.6 , respectively, which were optimized so that the maximum similarity index (Eq. (6) in Subsection 2.5) could be obtained. The time interval for the partial differential equation was set as 0.1 .

\subsubsection{Addition of MS regions detected by a CAD system onto "tentative" white matter regions}

MS candidate regions, which were automatically detected in the FLAIR image by a CAD system for MS developed by Yamamoto et al. [25], were added onto the "tentative" white matter regions, because several high-contrast MS lesions were not included in the white matter regions. Figure 6 shows an illustration of addition of MS regions detected by a CAD system for MS. As shown in this figure, the holes corresponding to MS regions in the "tentative" white matter regions were filled in by adding of the MS regions detected by a CAD system for MS.

Prior to the addition of MS regions, a morphological dilation operation with a $3 \times 3$-square kernel was applied to the MS candidate regions. At the end of this processing, a morphological closing operation with a $3 \times 3$-square kernel was applied three times to white matter candidate regions with MS candidate regions for smoothing of candidate regions.

The overall scheme for segmentation of MS regions is shown in Fig. 7. In the CAD system [25], MS candidate regions were detected through the following steps:

(1) MS lesions were enhanced by subtraction of a background image, which was approximated by the first order polynomial in the brain parenchymal region from the FLAIR image. (2) The initial candidates were identified by use of a multiple gray-level thresholding technique on the subtraction image as the points with local maximum pixel values [32,33]. MS candidate regions 
were segmented by use of a region-growing technique from the location of the initial candidates based on monitoring of large changes in five image features, i.e., effective diameter, area, circularity, slenderness, and the difference in the mean pixel value within the inner and outer regions of a candidate region. (3) The large number of false positive regions was reduced based on a rule-based method. (4) Final regions in MS candidates were determined by use of a level set method, which was used for reduction of false positives as well as more accurate segmentation. (5) All candidate regions were classified into true positive and false positive candidate regions by use of a support vector machine, which is a classifier based on a statistical learning theory.

\subsubsection{Removing of basal ganglia and thalamus from white matter candidate regions}

Final white matter regions were determined by removal of a basal ganglia and thalamus template of the gray matter from the white matter regions, because it was difficult to remove the basal ganglia and thalamus regions of the gray matter from the white matter regions due to the very low contrast. The basal ganglia and thalamus template shown in Fig. 8 was produced manually from a T2-weighted image of one patient out of the 14 cases used in this study. The slices including the basal ganglia and thalamus were selected manually, and then the basal ganglia and thalamus template was adjusted to each brain parenchyma by use of a $2 \mathrm{D}$ affine transformation [34]. Finally, the white matter regions were determined after removal of the adjusted template from the tentative white matter regions. The nine feature points for the affine transformation were selected automatically on two straight lines that ran at right angles to one another, in the circumscribed rectangle of the brain parenchyma. 
capsules in the T1-weighted, T2-weighted, and FLAIR images. Therefore, the internal capsule was included in the gold standard regions of gray matter in this study.

\subsection{Segmentation of gray matter regions}

The gray matter regions were obtained by subtraction of the white matter regions from the brain parenchymal region.

\subsection{Evaluation of segmentation accuracy}

The segmentation accuracy of our method was evaluated by use of a similarity index [35], which means the degree of similarity between the candidate region $C$ obtained by our method and the gold standard region $G$ obtained by a manual method. The similarity index was obtained by the following equation:

Similarity index $(\%)=\frac{2 n(G \cap C)}{n(G)+n(C)} \times 100$,

where $n(G)$ was the number of gold standard pixels, $n(C)$ was the number of segmented pixels automatically determined by use of our method, and $n(G \cap C)$ was the number of logical AND pixels between $G$ and $C$. We defined the gold standard regions based on manual contouring by an experienced neuroradiologist, following verification by a senior experienced neuroradiologist. The gold standard regions of the brain parenchyma and white matter were determined by the neuroradiologist's delineating their contours on the T1-weighted image and T2-weighted image, respectively. Moreover, the gold standard regions of gray matter were obtained by subtraction of the white matter regions from brain parenchymal regions. Therefore, we evaluated the segmentation accuracy of the brain parenchymal and white matter regions by 
using the similarity index. However, the variability of the gold standard was not investigated in this study, but is clarified in the Discussion Section.

\section{Results}

(1)

We investigated the computational impact of the CAD step on the proposed method. Results were obtained by use of a personal computer with two $2.66 \mathrm{GHz}$ Intel Dual-Core Xeon CPUs and 5 GB memory. It took about 20 seconds and 150 seconds on average for the segmentation step and the CAD step to deal with each MR image, respectively. Therefore, the computational impact of the CAD step on the proposed method was $88 \%$ on average.

Table 1 shows the average similarity indices of all steps for the white matter regions. The average similarity indices for white matter regions without and with addition of MS candidate regions to the "tentative" white matter regions were $80.3 \pm 10.3 \%$ and $80.5 \pm 10.5 \%$, respectively, in MS patients. According to these average results, the addition of MS candidate regions does not seem to be effective for accurate segmentation of the white matter regions. However, Fig. 9 shows a good example of the effect of adding MS candidate regions, i.e., segmented white matter regions of an MS patient without and with adding of MS candidate regions. The similarity index for the white matter regions increased from $84.7 \%$ to $89.3 \%$ by use of the proposed CAD system. The average similarity index for white matter regions increased from $80.5 \pm 10.5 \%$ to $85.2 \pm 4.3 \%$ in MS patients by removal of the basal ganglia and thalamus template region from the "tentative" white matter regions. Figure 10 shows two images, which are the white matter regions of an MS patient without and with removal of the basal ganglia and thalamus template region, respectively. The similarity index for the white matter regions increased from $68.2 \%$ to $84.0 \%$. Furthermore, the average similarity index for white matter regions increased from $81.0 \pm 9.8 \%$ to $85.9 \pm 3.4 \%$ in the control subjects, and 
increased from $80.7 \pm 9.9 \%$ to $85.5 \pm 3.8 \%$ in all cases by removal of the basal ganglia and thalamus template region.

As a final result, Fig. 11 shows the similarity indices for white matter and gray matter regions of all slices, and Table 2 shows the average similarity indices for the brain parenchymal and white matter regions. The average similarity indices of brain parenchymal and white matter regions were $95.5 \pm 1.2 \%$ and $85.2 \pm 4.3 \%$, respectively, for MS patients. Moreover, they were $95.0 \pm 2.0 \%$ and $85.9 \pm 3.4 \%$, respectively, for the control subjects. Here, there were no significant differences in the segmentation accuracy of any regions between MS patients and controls $(P>0.35)$. In all cases, the average similarity index was $95.2 \pm 1.6 \%$ for brain parenchymal regions and $85.5 \pm 3.8 \%$ for white matter regions. Examples of regions segmented by the proposed method are shown in Fig. 12. The similarity index was $95.9 \%$ for the brain parenchymal region and $85.7 \%$ for white matter regions.

\section{Discussion}

The proposed method is based on three kinds of 2D MR images, because (1) the T2-weighted and/or FLAIR 2D images have been established as routine sequences for diagnosis of MS lesions [36-38], (2) the data acquisition time of a 2D MR image is shorter than that of a 3D image, and (3) the in-plane spatial resolution and contrast in a 2D MR image can be higher than those of a 3D image, respectively. Nevertheless, there are a number of advantages of 3D imaging for accurate diagnosis of MS, such as identification of 3D locations of MS lesions and more accurate segmentation. The 3D locations of MS lesions are associated with visual, motor, and sensory impairments. Therefore, we plan to modify the proposed method from the 2D-based method to a 3D-based one.

The segmentation accuracy depends on the strength of the magnetic field. In this study, 

strength increases the image noise, which could lead to inaccurate segmentation results. Therefore, we should investigate the robustness of the proposed method by applying it to MR images acquired on 1.5 Tesla or lower field MR systems in future work. regions as well as final white matter regions. As a result, however, there were 47 false positive regions in all 42 slices used in this study, and thus the area ratio of the false positive regions to the gold standard white matter regions was $0.17 \pm 0.44 \%$ on average for each slice. Although the number of false positive regions should be reduced as much as possible, there is little impact of false positives on the segmentation accuracy in the proposed method. overestimated by the proposed method at each step of the segmentation of white matter regions. For that purpose, we calculated an overlap fraction (OF) and extra fraction (EF) [39], which can evaluate underestimated and overestimated regions, respectively. The OF and EF are defined as

$$
\text { Extra fraction }(\%)=\frac{F P}{T P+F N} \times 100
$$

where TP, FP, and FN are true positive, false positive, and false negative pixels, respectively.

The OF approaches unity with decreasing underestimated regions, whereas the EF approaches zero with decreasing overestimated regions. For MS patients, the average OFs for white matter regions without and with addition of CAD outputs were $89.2 \pm 6.1 \%$ and $90.5 \pm 5.1 \%$, respectively, and the average EFs were $35.7 \pm 27.8 \%$ and $37.6 \pm 29.1 \%$, respectively. In this step, some MS regions were removed as FNs, but also some FP regions were added onto the white matter regions. Furthermore, for MS patients, the average OFs for white matter regions without and with removal of the basal ganglia and thalamus regions were $90.5 \pm 5.1 \%$ and 89.8 
$\pm 5.4 \%$, respectively, and the average EF decreased from $37.6 \pm 29.1 \%$ to $21.1 \pm 8.7 \%(P<$ 0.05). On the other hand, for control subjects, the average OFs without and with removal of the basal ganglia and thalamus regions were $88.7 \pm 3.7 \%$ and $88.2 \pm 4.3 \%$, respectively, and the average EF decreased from $33.0 \pm 26.2 \%$ to $17.4 \pm 7.1 \%(P<0.05)$. Consequently, the average OF and EF in all cases were $89.0 \pm 4.9 \%$ and $19.2 \pm 8.1 \%$, respectively, in the final step of the segmentation of white matter regions.

Although the gold standard regions for the brain parenchyma, white matter, and gray matter regions were based on manual contouring with the consensus of two experienced neuroradiologists in this study, it is important to consider inter- and intra-observer variability when the gold standard regions are determined by manual segmentation. Gao et al. [40] reported that there was intra-observer variability (maximum SD ranging from $2 \%$ to $8 \%$ of the mean) and inter-observer variability (SD of the observers' means being $18.8 \%$ of the mean volume) in the manual delineation of prostate volume on a computed tomography (CT) image for radiation therapy. The variability in the delineation of the white matter and gray matter could be larger than that of the prostate due to their complicated shapes. Therefore, we should investigate the variability of manual segmentation for white matter and gray matter regions by several observers, and then calculate the similarity index by considering the variability of the gold standards in future work.

Other limitations of this study need to be described. First, the CAD system for detection of MS regions [25] produced a few false positives, and it was not able to detect a number of MS regions. According to a report by Yamamoto et al. [25], the sensitivity and the number of false positives were $81.5 \%$ and 2.9 , respectively, for 3 MS cases including $168 \mathrm{MS}$ lesions, two cases of which were used for this study. The false positives and false negatives could lead to the overestimation and underestimation of white matter regions, respectively. Therefore, the CAD system should be improved in terms of the detection accuracy. Second, we 
did not deal with cases where MS lesions were developed in the gray matter regions which were

400

401

402

403

404

405

406

407

408

409

410

411

412

413

414

415

416

417

418 reported by Kidd et al. [41] and Peterson et al. [42]. However, the current proposed method considers all MS lesions as a part of the white matter regions [1-8], because the majority of MS lesions develop in the white matter regions. Nonetheless, we should improve the proposed method so that MS lesions detected by the CAD system can be classified correctly in the white matter and gray matter regions.

\section{Conclusions}

We have developed an automated method for segmentation of the white matter and gray matter regions including the MS lesions. As a result, the white matter and gray matter regions are segmented automatically even if patients have MS lesions. Therefore, our proposed method might be feasible as a diagnostic tool for MS patients in clinical practice.

\section{Acknowledgments}

The authors are grateful to Dr. Toshiaki Miyachi, Kanazawa University, for useful suggestion, Dr. Junji Morishita, and Dr. Seiji Kumazawa, Kyushu University, for helpful discussion. 


\section{References}

420

421

422

423

424

425

426

427

428

429

430

431

432

433

434

435

436

1. Losseff NA, Wang L, Lai HM, Yoo DS, Gawne-Cain ML, McDonald WI, et al. Progressive cerebral atrophy in multiple sclerosis. A serial MRI study. Brain. 1996;119:2009-19.

2. Ge Y, Grossman RI, Udupa JK, Babb JS, Nyul LG, Kolson DL. Brain atrophy in relapsing-remitting multiple sclerosis: fractional volumetric analysis of gray matter and white matter. Radiology. 2001;220:606-10.

3. Chard DT, Griffin CM, Parker GJ, Kapoor R, Thompson AJ, Miller DH. Brain atrophy in clinically early relapsing-remitting multiple sclerosis. Brain. 2002;125:327-37.

4. Quarantelli M, Ciarmiello A, Morra VB, Orefice G, Larobina M, Lanzillo R, et al. Brain tissue volume changes in relapsing- remitting multiple sclerosis: correlation with lesion load. NeuroImage. 2003;18:360-6.

5. De Stefano N, Matthews PM, Filippi M, Agosta F, De Luca M, Bartolozzi ML, et al. Evidence of early cortical atrophy in MS Relevance to white matter changes and disability. Neurology. 2003;60:1157-62.

6. Dalton CM, Chard DT, Davies GR, Miszkiel KA, Altmann DR, Fernando K, et al. Early development of multiple sclerosis is associated with progressive grey matter atrophy in patients presenting with clinically isolated syndromes. Brain. 2004;127:1101-7.

7. Carone DA, Benedict RH, Dwyer MG, Cookfair DL, Srinivasaraghavan B, Tjoa CW, et al. 
437 Semi-automatic brain region extraction (SABRE) reveals superior cortical and deep gray

438 matter atrophy in MS. NeuroImage. 2006;29:505-14.

439 8. Sastre-Garriga J, Ingle GT, Chard DT, Ramio-Torrenta L, Miller DH, Thompson AJ. Grey

440 and white matter atrophy in early clinical stages of primary progressive multiple sclerosis.

$441 \quad$ NeuroImage. 2004;22:353-9.

442 9. Sanfilipo MP, Benedict RH, Sharma J, Weinstock-Guttman B, Bakshi R. The relationship

443 between whole brain volume and disability in multiple sclerosis: a comparison of normalized

444 gray vs. white matter with misclassification correction. NeuroImage. 2005;26:1068-77.

445 10. Udupa JK, Samarasekera S. Fuzzy connectedness and object definition: theory, algorithms,

446 and applications in image segmentation. Graphical Models Image Process. 1996;58:246-61.

447 11. Alfano B, Brunetti A, Covelli EM, Quarantelli M, Panico MR, Ciarmiello A, et al.

448 Unsupervised, automated segmentation of the normal brain using a multispectral relaxometric

449 magnetic resonance approach. Magn Reson Med. 1997;37:84-93.

450 12. Ashburner J, Friston KJ. Voxel-based morphometry--the methods. NeuroImage.

$451 \quad 2000 ; 11: 805-21$.

452 13. Liu T, Li H, Wong K, Tarokh A, Guo L, Wong ST. Brain tissue segmentation based on DTI

453 data. NeuroImage. 2007;38:114-23.

454 14. Vrooman HA, Cocosco CA, van der Lijn F, Stokking R, Ikram MA, Vernooij MW, et al. 

classification. NeuroImage. 2007;37:71-81.

15. Hu Q, Qian G, Teistler M, Huang S. Informatics in radiology: automatic and adaptive brain morphometry on MR images. Radiographics. 2008;28:345-56.

16. Lee H, Prohovnik I. Cross-validation of brain segmentation by SPM5 and SIENAX. Psychiatry Res. 2008;164:172-7.

17. Chao WH, Chen YY, Lin SH, Shih YY, Tsang S. Automatic segmentation of magnetic resonance images using a decision tree with spatial information. Comput Med Imaging Graph. $2009 ; 33: 111-21$

18. Klauschen F, Goldman A, Barra V, Meyer-Lindenberg A, Lundervold A. Evaluation of automated brain MR image segmentation and volumetry methods. Hum Brain Mapp. 2009;30:1310-27.

19. Lee JD, Su HR, Cheng PE, Liou M, Aston JA, Tsai AC, et al. MR image segmentation using a power transformation approach. IEEE Trans Med Imaging. 2009;28:894-905.

20. Smith SM, De Stefano N, Jenkinson M, Matthews PM. Normalized accurate measurement of longitudinal brain change. J Comput Assist Tomogr. 2001;25:466-75. 

with multiple sclerosis. J Magn Reson Imaging. 2000;12:799-807. Computer-aided evaluation method of white matter hyperintensities related to subcortical vascular dementia based on magnetic resonance imaging. Comput Med Imaging Graph. 2010;34:370-6.

23. Otsu N. A threshold selection method from gray-level histograms. IEEE Trans Syst Man Cybern. 1979;9:62-6.

24. Sethian JA. Level set methods and fast marching methods: evolving interfaces in 
28. Yui S, Hara K, Zha H, Hasegawa T. A fast narrow band method and its application in topology-adaptive 3-D modeling. Int Conf Patt Recogn. 2002;4:122-5. method and its real-time applications. IEEE Int Conf on Syst Man Cybern. 2004;7:6302-7. acute cerebral infarction. Int J CARS. 2007;2:105-15.

31. Nagao M, Matsuyama T. Edge preserving smoothing. Comput Graph Image Process. 1979;9:394-407.

501 for automated detection of lung nodules in low-dose computed tomography images for lung $502 \quad$ cancer screening. Acad Radiol. 2004;11:617-29. to subcortical vascular dementia on magnetic resonance images. Acad Radiol. 2008;15:978-85. 
510 Recommended diagnostic criteria for multiple sclerosis: Guidelines from the International

511 Panel on the Diagnosis of Multiple Sclerosis. Ann Neurol. 2001;50:121-7.

512 37. Filippi M, Rocca MA, Arnold DL, Bakshi R, Barkhof F, De Stefano N, et al. EFNS

513 guidelines on the use of neuroimaging in the management of multiple sclerosis. Eur J Neurol.

$514 \quad 2006 ; 13: 313-25$.

38. Simon JH, Li D, Traboulsee A, Coyle PK, Arnold DL, Barkhof F, et al. Standardized MR

516 imaging protocol for multiple sclerosis: Consortium of MS centers consensus guidelines. Am

$517 \quad$ J Neuroradiol. 2006;27:455-61.

39. Stokking R, Vincken KL, Viergever MA. Automatic morphology-based brain segmentation (MBRASE) from MRI-T1 data. NeuroImage. 2000;12:726-38.

40. Gao Z, Wilkins D, Eapen L, Morash C, Wassef Y, Gerig L. A study of prostate delineation

521 referenced against a gold standard created from the visible human data. Radiother Oncol. 2007;85:239-46. multiple sclerosis. Brain. 1999;122:17-26.

42. Peterson JW, Bo L, Mork S, Chang A, Trapp BD. Transected neurites, apoptotic neurons, and reduced inflammation in cortical multiple sclerosis lesions. Ann Neurol. 

2001;50:389-400.

528 
529 Table 1 Average similarity indices of all steps for white matter regions.

530

\begin{tabular}{cccc}
\hline & MS patients (\%) & Controls (\%) & All cases (\%) \\
\hline Initial white matter & $80.3 \pm 10.3$ & $81.0 \pm 9.8$ & $80.7 \pm 9.9$ \\
Level set method & $80.3 \pm 10.3$ & $81.0 \pm 9.8$ & $80.7 \pm 9.9$ \\
Addition of MS regions & $80.5 \pm 10.5$ & - & - \\
Removal of BGT template & $85.2 \pm 4.3$ & $85.9 \pm 3.4$ & $85.5 \pm 3.8$ \\
\hline
\end{tabular}

531

532

533 
534 Table 2 Average similarity indices between regions obtained by the proposed method and 535 neuroradiologists for brain parenchymal, white matter, and gray matter regions.

536

\begin{tabular}{ccccc}
\hline & MS patients (\%) & Controls (\%) & $P$ value & All cases (\%) \\
\hline Brain parenchyma & $95.5 \pm 1.2$ & $95.0 \pm 2.0$ & 0.359 & $95.2 \pm 1.6$ \\
White matter & $85.2 \pm 4.3$ & $85.9 \pm 3.4$ & 0.572 & $85.5 \pm 3.8$ \\
\hline
\end{tabular}

537

538 
540 Fig. 1 Pixel value histogram of an original T1-weighted image, which has four parts

541 corresponding to the background, CSF, brain parenchymal, and fat regions, respectively. $T_{B G}$,

$542 T_{C S F}$ and $T_{F A T}$ are the threshold values for reducing the background, CSF, and fat regions,

543 respectively.

544

545 Fig. 2 Overall scheme for segmentation of white matter (WM) regions.

546

Fig. 3 Pixel value histograms of a T2-weighted image (T2WI), a T1-weighted image (T1WI), and a T2-T1 subtraction image in the brain parenchymal region.

Fig. 4 Brain parenchymal regions in a T2-weighted image, b T1-weighted image, and $\mathbf{c}$ T2-T1 subtraction image.

Fig. 5 Comparison of results obtained by three smoothing filters: $\mathbf{a}$ an original image, $\mathbf{b}$ with a

554 Gaussian filter, $\mathbf{c}$ with an edge-preserving smoothing filter, and $\mathbf{d}$ with an adaptive partial median filter. 
557 Fig. 6 Illustration of addition of MS regions detected by a CAD system for MS onto "tentative"

558 white matter regions: a MS candidate regions detected by a CAD system, b "tentative" white

559 matter regions, where there were holes corresponding to MS regions, c "tentative" white matter

560 regions, where the holes were filled in by adding of the MS regions.

561

Fig. 7 Overall scheme for segmentation of MS regions.

563

564

Fig. 8 A template with basal ganglia and thalamus.

565

Fig. 9 Effect of adding MS candidate regions obtained by a CAD system on segmentation of the

white matter regions indicated by white lines: $\mathbf{a}$ without and $\mathbf{b}$ with addition of MS candidate

regions obtained by the CAD system, and $\mathbf{c}$ corresponding gold standard regions. The similarity

Fig. 10 Effect of removing basal ganglia and thalamus from the white matter regions indicated by white lines: a without and $\mathbf{b}$ with removal of the basal ganglia and thalamus regions, and $\mathbf{c}$ corresponding gold standard regions. The similarity index for the white matter regions increased 
576 Fig. 11 Relationship between the average similarity indices for white matter and gray matter

577 regions.

578

579 Fig. 12 Illustrations of brain parenchymal region (a, d), white matter regions $(\mathbf{b}, \mathbf{e})$, and gray

580 matter regions $(\mathbf{c}, \mathbf{f})$. White lines indicate output regions by the proposed method $(\mathbf{a}, \mathbf{b}, \mathbf{c})$ and corresponding gold standard regions (d, e, f). The similarity index was 95.9\% for brain parenchymal region and $85.7 \%$ for white matter regions.

583 
Fig. 1

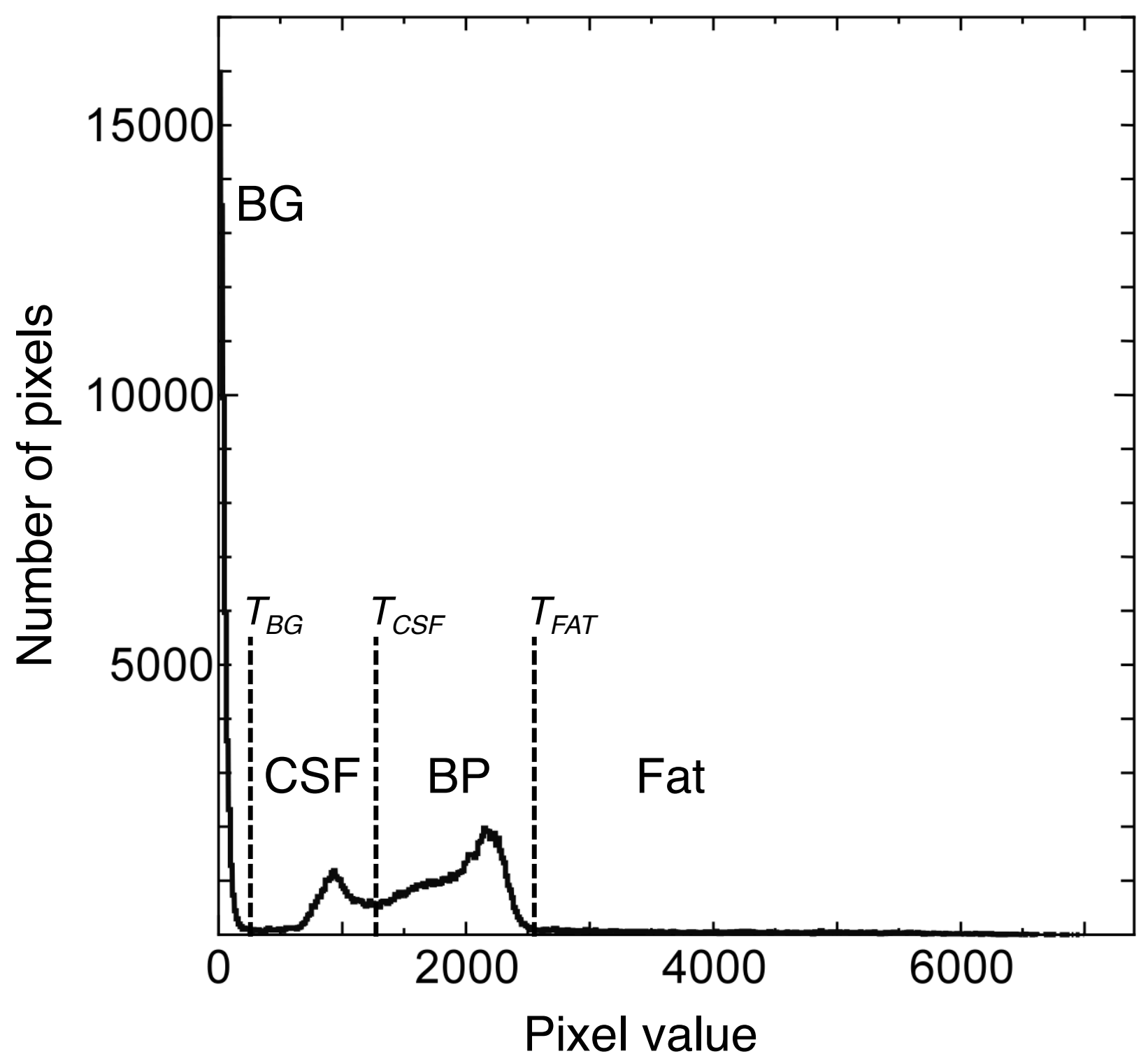


T1-, T2-weighted and FLAIR images

Segmentation of brain parenchymal region

T2 - T1 subtraction image

Segmentation of "tentative" WM regions

based on a level set method

Addition of MS regions detected by

a CAD system onto "tentative" WM regions

Removing of a BGT template from

"tentative" WM regions

Final white matter regions 
Fig. 3

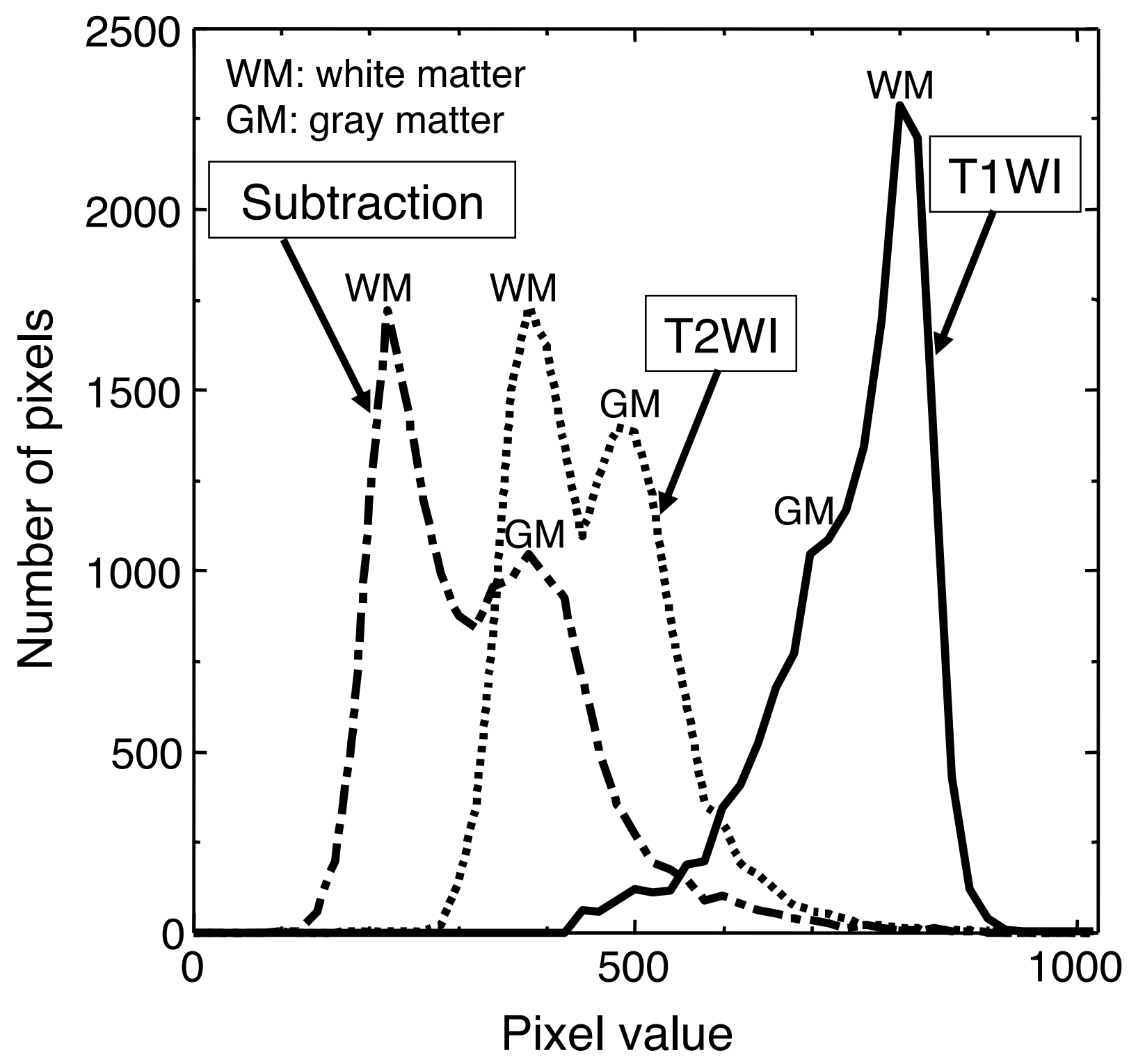


Fig. 4
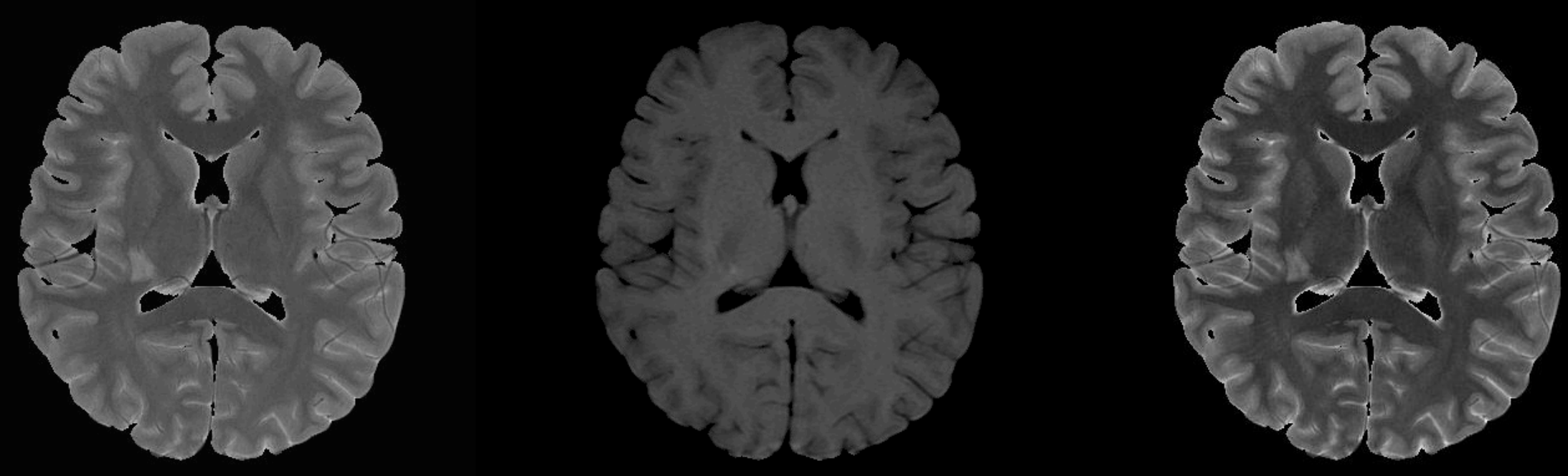

(a)

(b)

(c) 


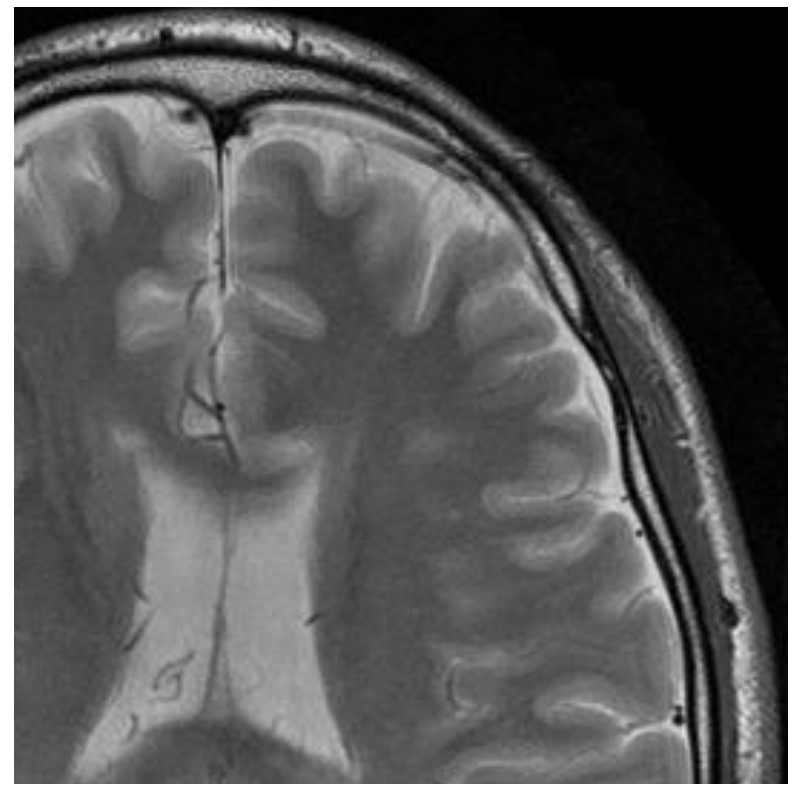

(a)

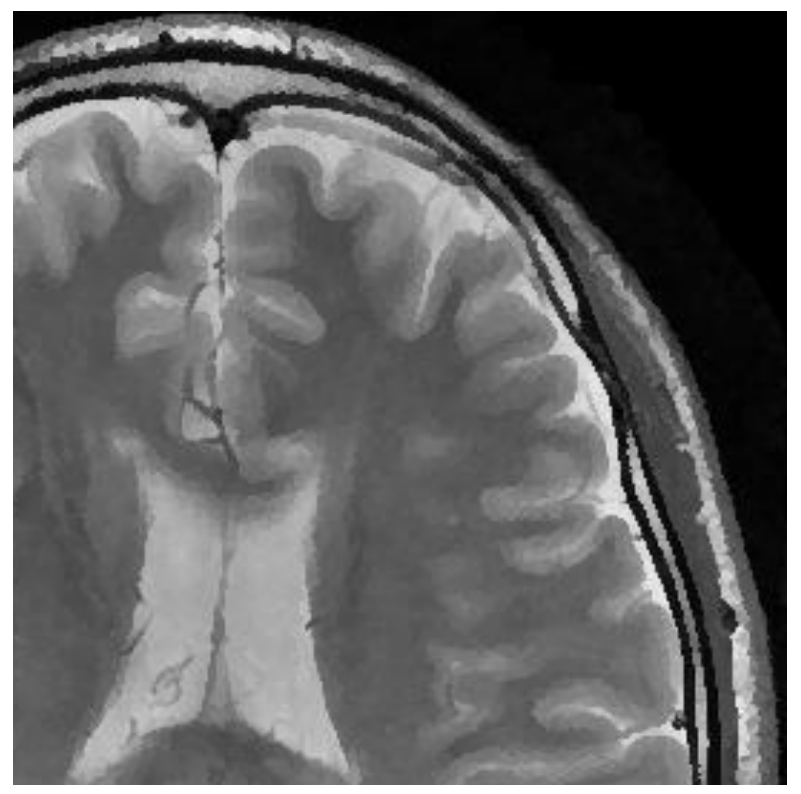

(c)

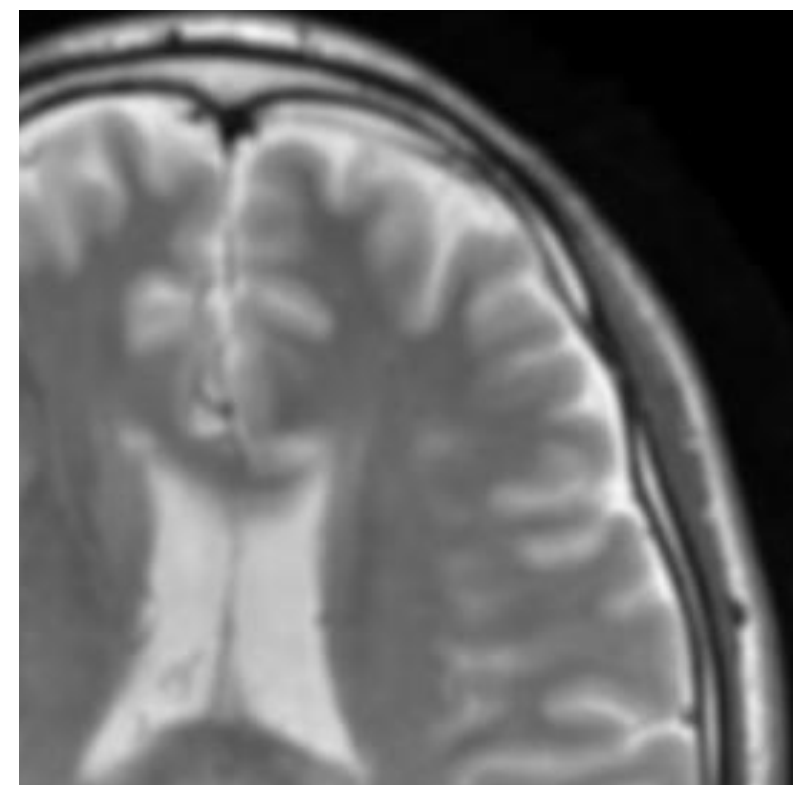

(b)

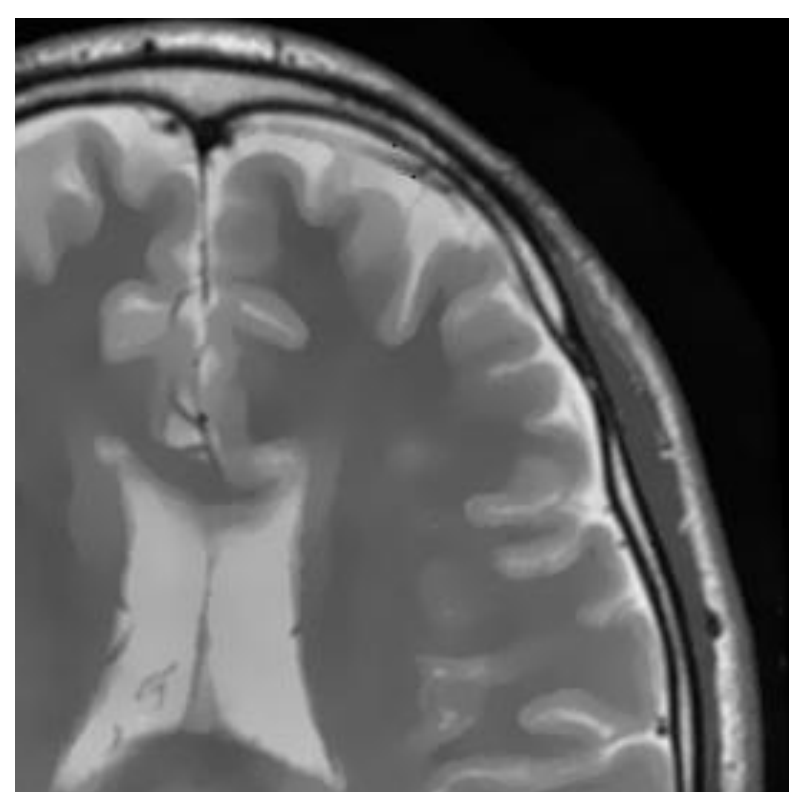

(d)

Fig. 5 
Fig. 6

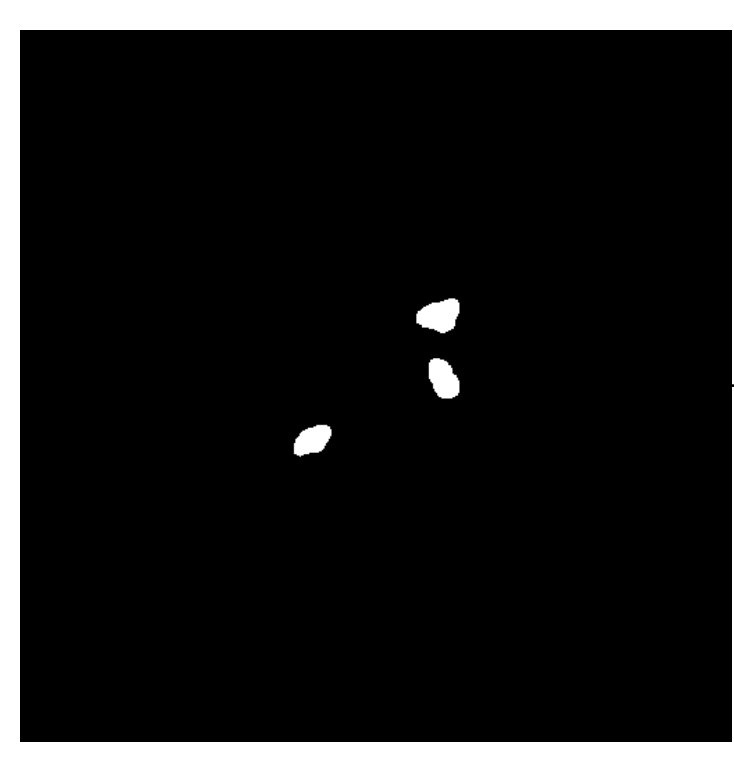

(a)

$$
\text { 位 }
$$

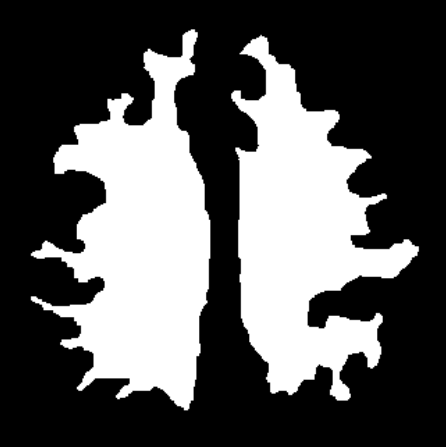

(c)

(b) 


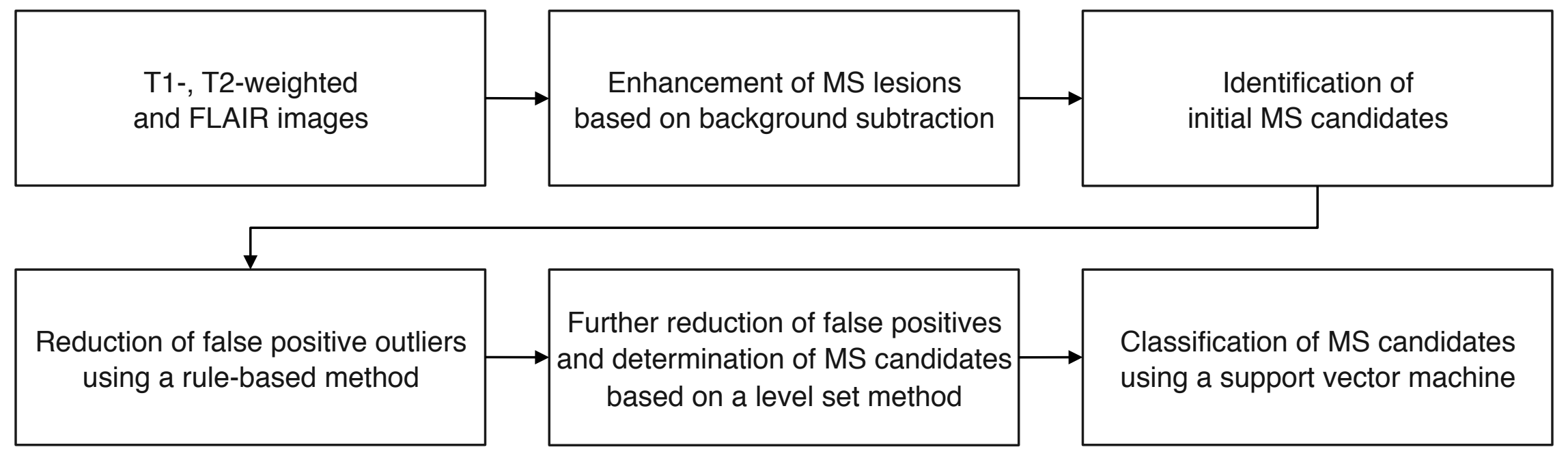


Fig. 8

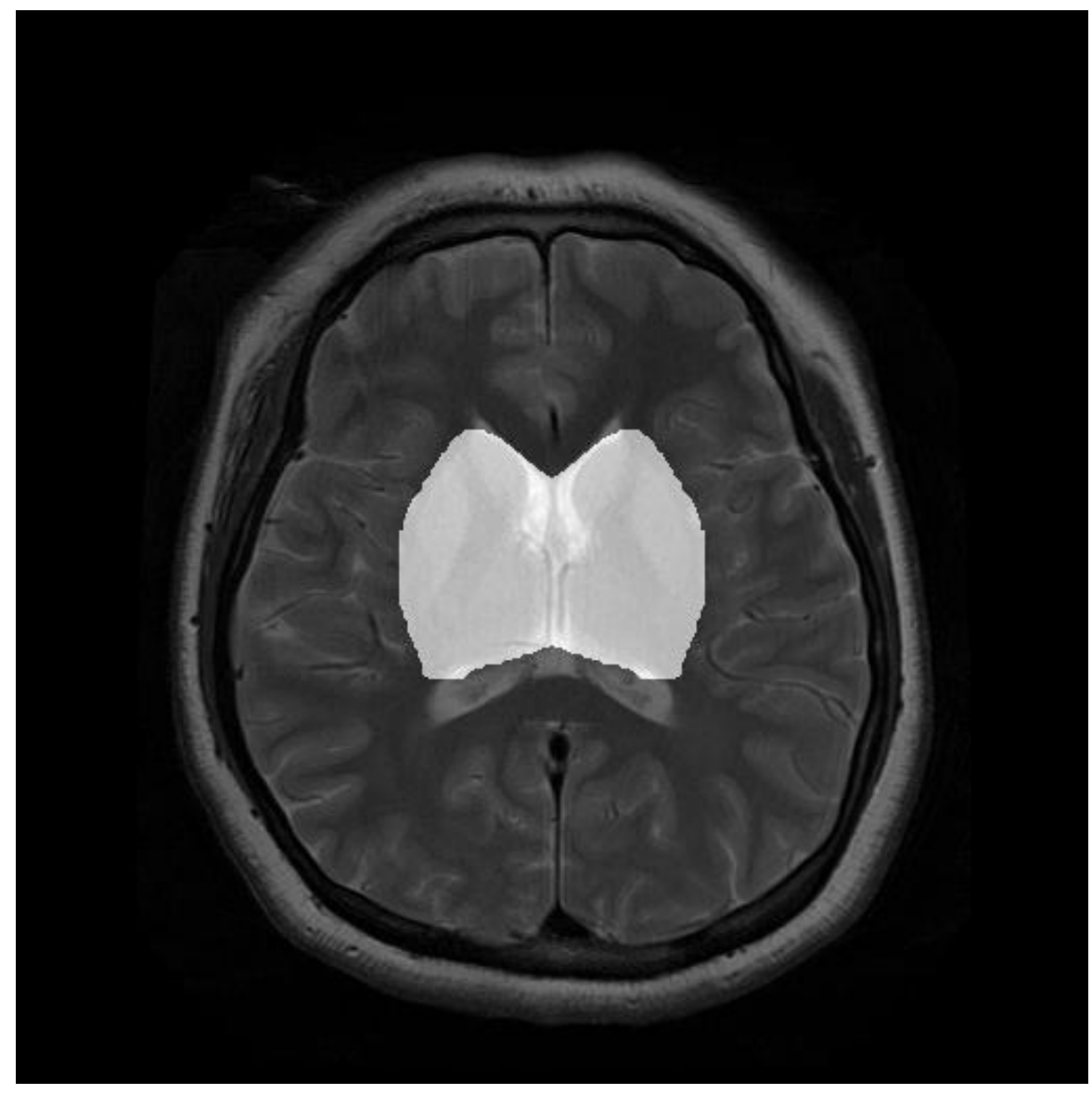


Fig. 9
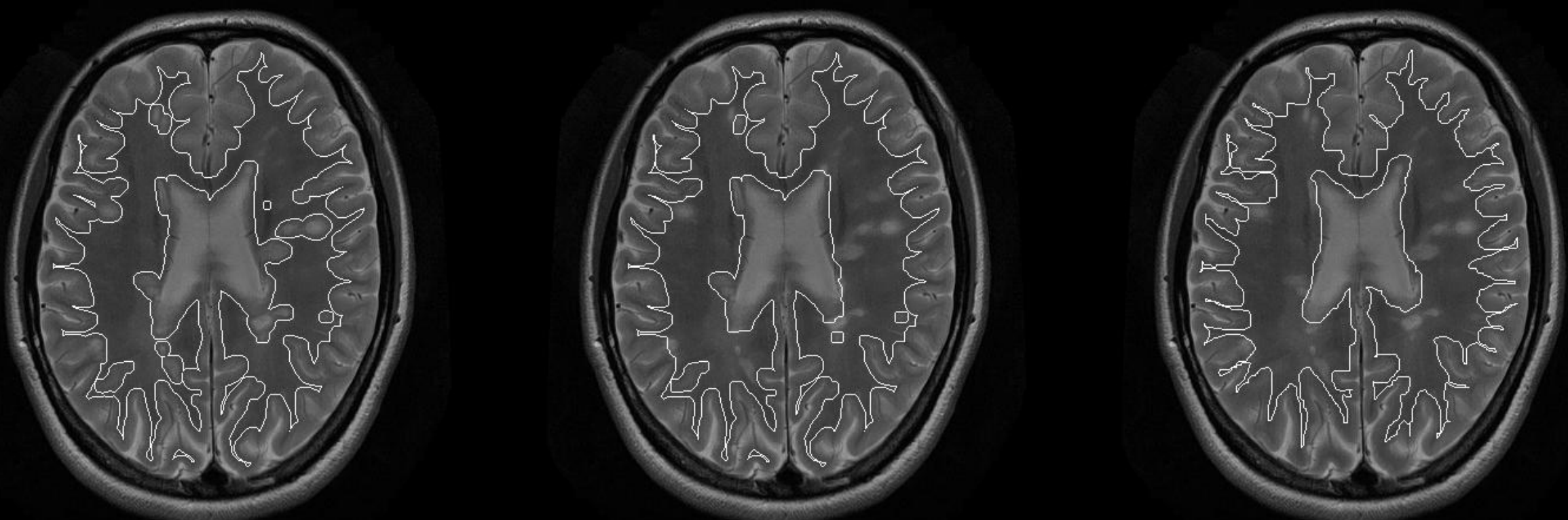

(a)

(b)

(c) 
Fig. 10
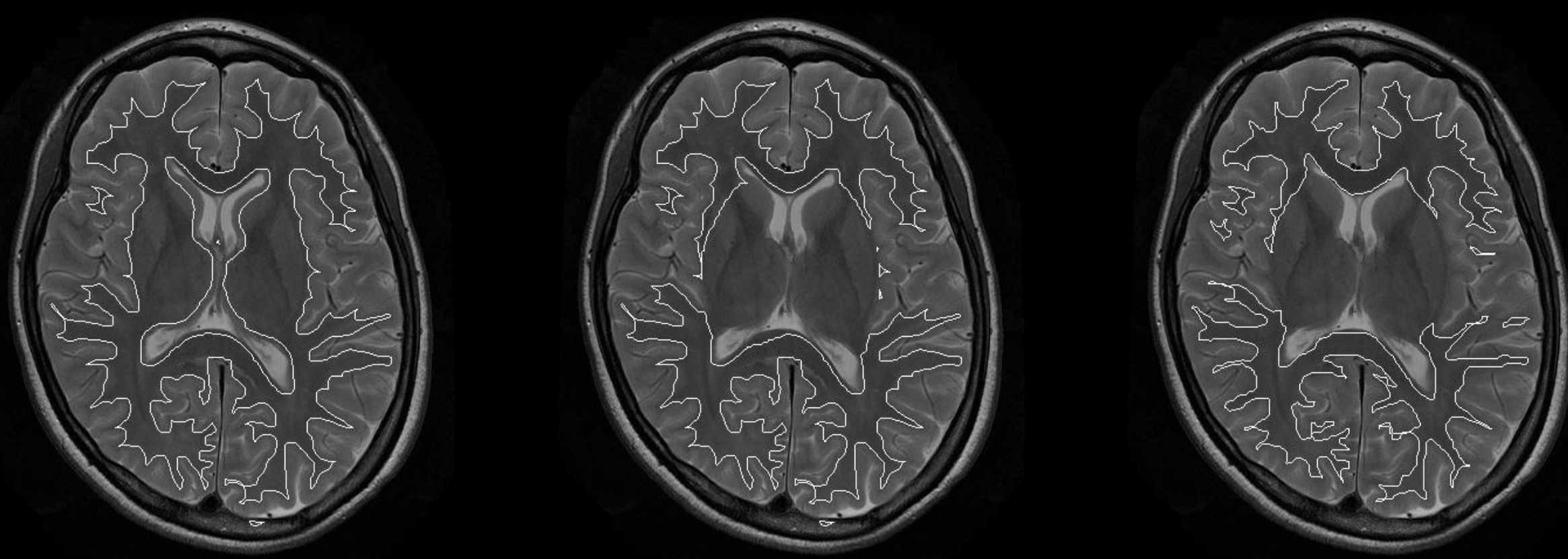

(a)

(b)

(c) 
Fig. 11

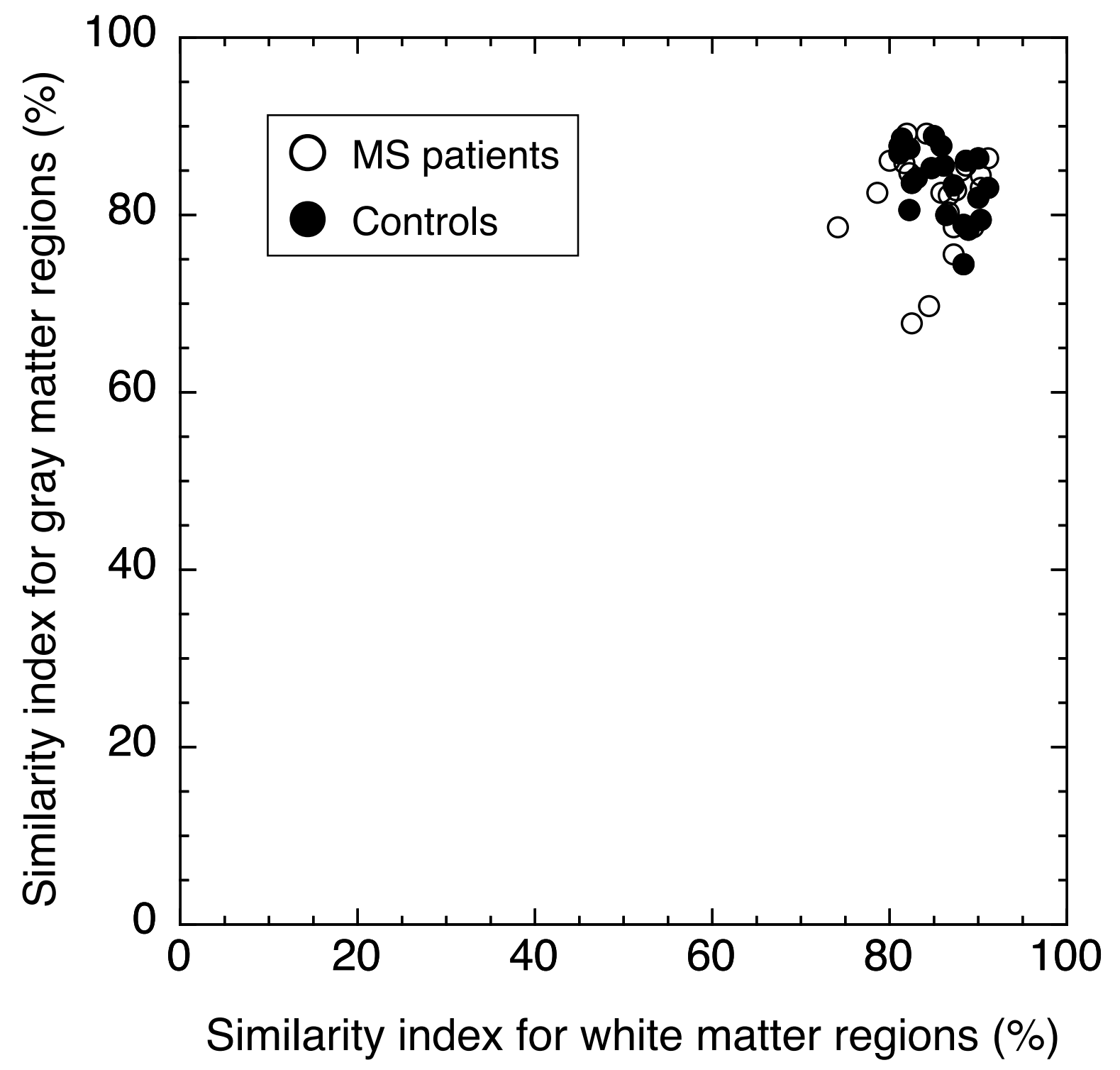




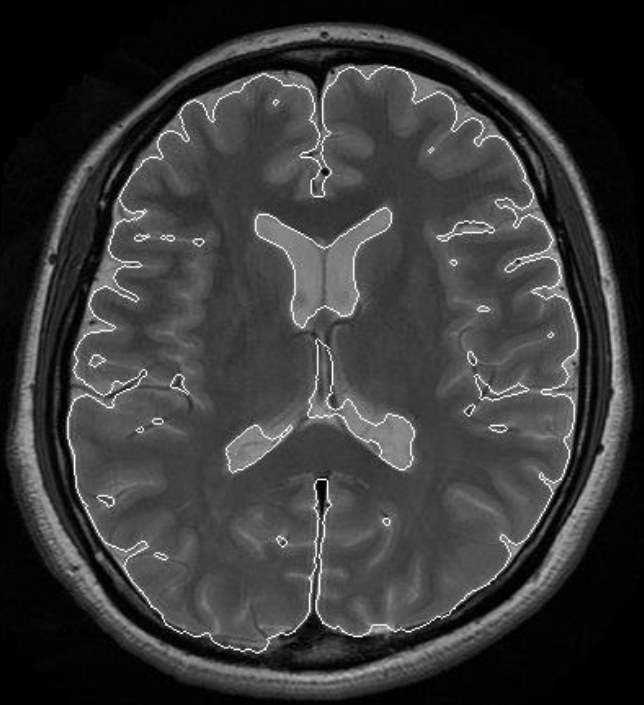

(a)

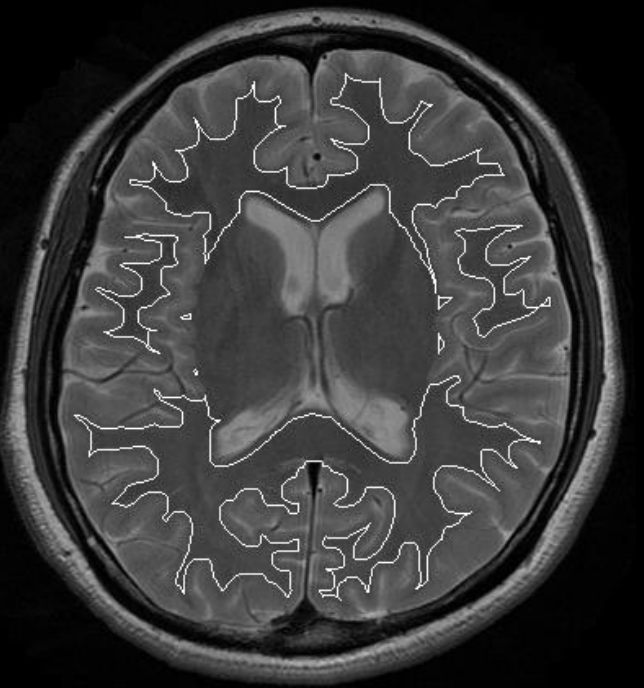

(b)

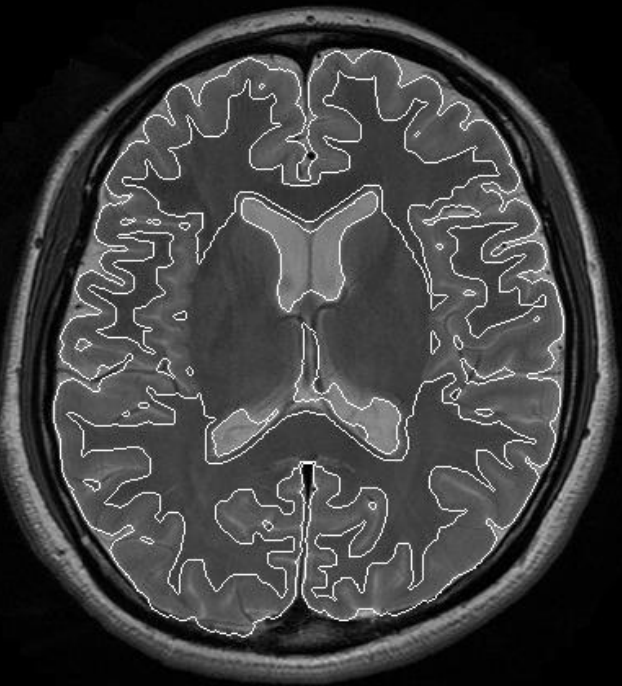

(c)

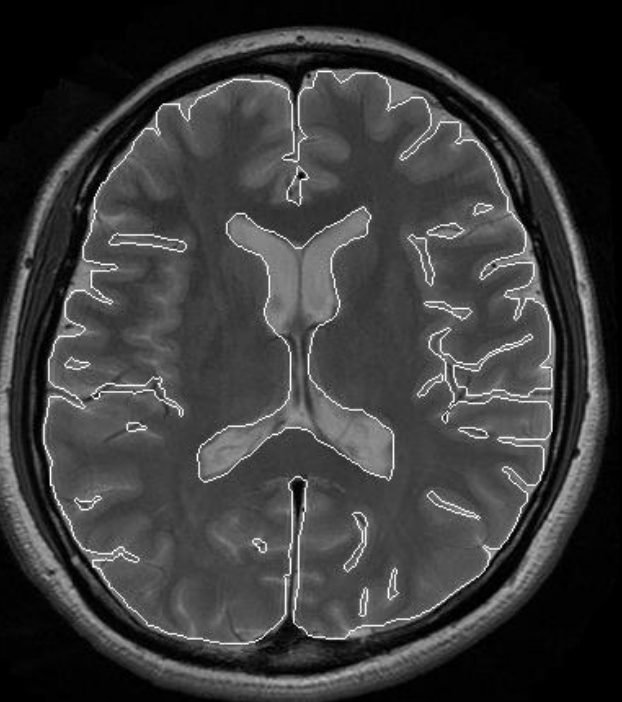

(d)

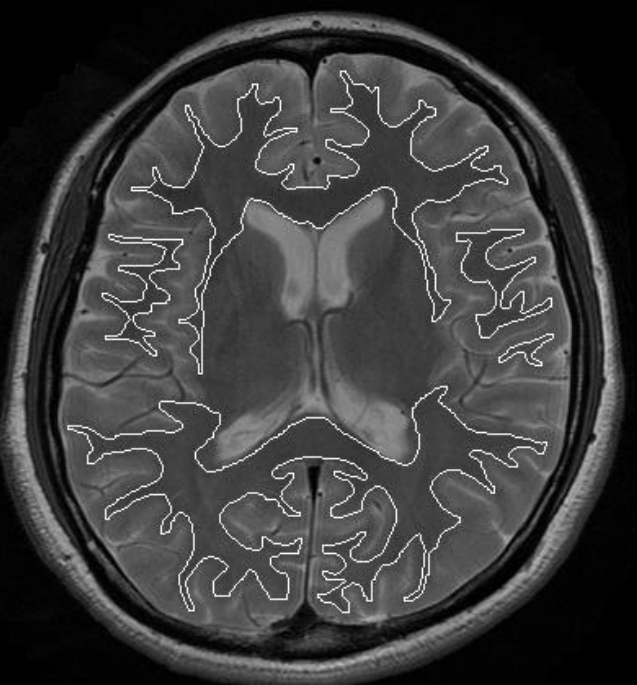

(e)

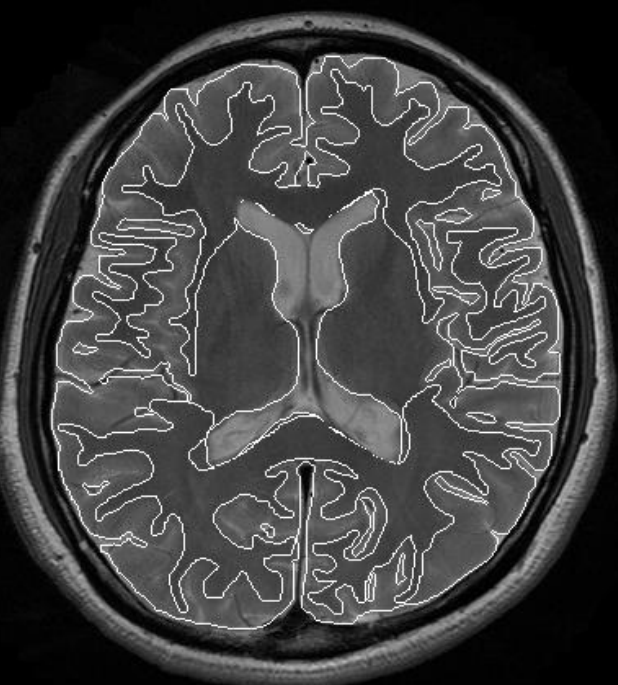

(f) 